Check for updates

Cite this: RSC Adv., 2017, 7, 51121

\title{
Real-time visualization of cardiac cell beating behaviour on polymer diffraction gratings $\uparrow$
}

\author{
A. Gibbons, (D) ab O. Lang, ${ }^{c}$ Y. Kojima, ${ }^{\text {ad }}$ M. Ito, ${ }^{a}$ K. Ono, ${ }^{e}$ K. Tanaka*ab \\ and E. Sivaniah iD *a
}

Cardiotoxicity is one of the major adverse effects of pharmaceuticals and new methods are sought for measuring cell properties with high-throughput. Here we report a flexible diffraction grating device fabricated from polydimethylsiloxane coated with platinum to make optically responsive cell culturing substrates. Cardiomyocytes from neonatal Sprague-Dawley rats were isolated and seeded upon the surface of the PDMS diffraction grating using fibronectin as the binding protein. On this substrate the cells formed a confluent layer and continued beating synchronously. By measuring the optical change of the PDMS substrate, the beating of the confluent layer could be monitored. Epinephrine was introduced to the cell environment and the change in beating frequency was measured and visualized. This flexible diffraction grating substrate provides a new way for remotely measuring cell beating properties during high-throughput testing and within miniaturized or confined device architectures.

Received 11th June 2017

Accepted 20th October 2017

DOI: $10.1039 / \mathrm{c} 7 \mathrm{ra06515a}$

rsc.li/rsc-advances

the cells, however, when fully submerged in a liquid medium the beating signal becomes difficult to detect as the cells lift from the surface. ${ }^{6}$ AFM based approaches have also been used to measure cardiomyocytes. Ossola et al. were able to combine patch-clamp and AFM techniques to measure the membrane current and forces applied by a contracting cell. ${ }^{8}$

Many cell detecting methods have used customized polymers and gels as a substrate, where the stiffness of the gel allows the substrate to be physically deformed by the beating cells and measurements can be made from changes of the substrate itself. One of the earliest yet still active examples of such methods is traction force microscopy (TFM). In TFM hydrogels are embedded with marker beads and used as a substrate for the cells. The deformation of the substrate is then measured by observing the displacement of the marker beads. $^{9-12}$ Hybrids of thin patterned polydimethylsiloxane (PDMS) films and cardiomyocyte sheets known as muscular thin films have been used both as actuators and as detection devices to analyze the contraction of cardiac cells. ${ }^{13-15}$ PDMS can also be molded to make microstructures that can be used to analyze the forces applied by cardiomyocytes. Micropillars and cantilevers that move and deflect as a result of beating cells have been successfully developed and analyzed through the molding of PDMS. ${ }^{16-22}$ A similar approach utilized cantilevers fabricated from polyimide layers sandwiching a layer of $\mathrm{Cr} / \mathrm{Au}$ with a PDMS coating for insulation. The sensor was able to measure the beating of cardiac monolayers through deflection of the cantilever. ${ }^{23}$ Another interesting approach that has been taken is to use coherent light and patterned PDMS substrates to create Moire diffraction patterns to detect the contraction force of cells. ${ }^{24}$

${ }^{a}$ Institute for Integrated Cell-Material Sciences, Kyoto University, Kyoto, Japan.
esivaniah@icems.kyoto-u.ac.jp; Fax: +8175 753 9820; Tel: +8175 7539865

${ }^{b}$ Department of Physics and Astronomy, Graduate School of Science, Kyoto University, Kyoto, Japan

Chemotaxis Research Group, Department of Genetics, Cell and Immunobiology, Semmelweis University, Budapest, Hungary

${ }^{d}$ Center for iPS Cell Research and Application (CiRA), Kyoto University, Kyoto, Japan ${ }^{e}$ Department of Cardiovascular Medicine, Graduate School of Medicine, Kyoto University, Kyoto, Japan

$\dagger$ Electronic supplementary information (ESI) available. See DOI: 10.1039/c7ra06515a 
The disadvantage, however, of many of these methods is that only a single cardiac cell or a small population of cells can be measured simultaneously. For large-scale observations, optical microscopes still offer the most convenient method for detecting beating cells. However such optical techniques still face obstacles to implementation within a lab-on-a-chip type applications.

The approach taken in this study is to develop a surface that can detect the beating pattern of cardiomyocytes over a large area. We accomplished this by fabricating soft PDMS diffraction gratings. Diffraction gratings are devices that reflect (and pass) incident light at different angles depending on its incident angle and wavelength, Fig. 1 . This behaviour is a result of light interfering with itself after interacting with repeating structures and is described by the diffraction equation, ${ }^{25}$

$$
n \lambda=d\left(\sin \theta_{i}+\sin \theta_{d}\right)
$$

where $n, \lambda, d, \theta_{i}$, and $\theta_{d}$ are the diffraction order, light wavelength, grating element spacing, incident angle, and diffraction angle respectively.

The principle behind our device is that cells contracting on the surface of a diffraction grating should change the local diffraction pattern and cause a change of local light intensity. Aggregated over the entire substrate surface, these changes cause a detectable change in the overall measured light. Compared to other techniques we expect that this technique will allow easier monitoring of beating cells over larger regions. Substrate stiffness is an important aspect of cardiomyocyte environment to consider when designing a substrate due to its effect on aspects of cell behaviour such as contraction stress, ${ }^{26,27}$ and cell maturation including alignment of microfibrils. ${ }^{26,28}$ The optimal elasticity for the physiological environment of the cardiomyocytes is considered to be between $10 \mathrm{kPa}$ and $20 \mathrm{kPa} .^{27,29}$ PDMS gels can be fabricated within this range of stiffnesses and have the potential to be used for a soft diffraction grating. The advantage of a soft diffraction grating is that small forces on the surface should cause detectable changes in the diffracted light.

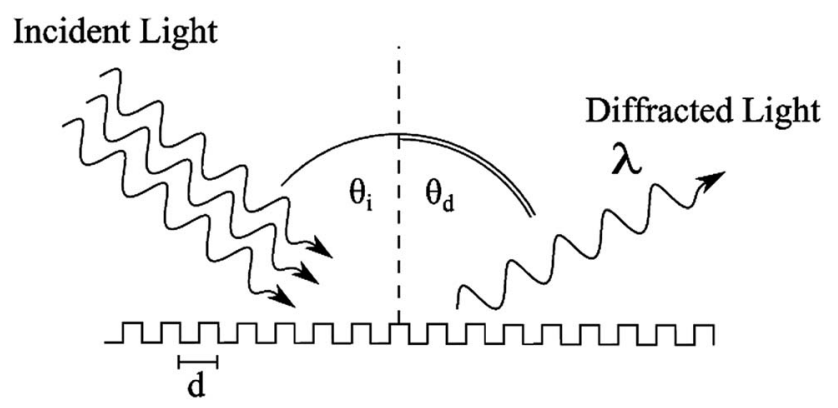

Fig. 1 Diagram of diffraction process. Incident light composed of multiple wavelengths of light undergoes diffraction when reflecting or transmitting through a repeating structure. The incident light comes in with an incident angle of $\theta_{i}$ and light with wavelength $\lambda$ is diffracted at angle $\theta_{d}$. The diffracted angle depends on the interval of the repeating structure, $d$, also referred to as the grating element spacing.

\section{Methodology}

\section{Preparation of PDMS diffraction gratings}

The PDMS diffraction gratings were made using Sylgard 184 Silicone Elastomer Kit from Dow Corning. The kit consists of a base and a crosslinker. A PDMS gel is prepared by mixing the crosslinker into the base and allowing the mixture to set over time.

The diffraction grating gels were prepared according to the scheme shown in Fig. 2. Blank CD disks (Maxwell CD-R, 700 $\mathrm{MB})$ were used as the diffraction pattern template. The pattern of the CD was exposed by removing the label from the top of the disk and pieces of appropriate size were cut out, for $53 \mathrm{~mm}$ Petri dishes $2 \mathrm{~cm}$ by $2 \mathrm{~cm}$ pieces were used. The choice of the CD pattern is a deliberate one, eschewing the use of more expensive optical elements as pattern templates, to simply highlight the accessibility of the proposed solution.

The PDMS gel was prepared by adding the crosslinker to the base, the weight ratio of crosslinker to base can be varied to control the stiffness of the PDMS. Gels with crosslinker: base ratios of $1: 30,1: 35,1: 40,1: 45$, and $1: 50$ were prepared having expected stiffnesses of around $100 \mathrm{kPa}, 50 \mathrm{kPa}, 20 \mathrm{kPa}, 15 \mathrm{kPa}$ and 9 $\mathrm{kPa}$ respectively, ${ }^{30}$ covering the physiologically relevant range of stiffnesses for cardiac cells. ${ }^{26,27}$ The crosslinker and base were thoroughly mixed by mechanical stirring for $5 \mathrm{~min}$. The mixture was then gently poured into $35 \mathrm{~mm}$ Petri dishes. To remove bubbles the mixture was degassed in a desiccator for $30 \mathrm{~min}$.

After the PDMS mixture levelled out in the Petri dishes, a CD piece was placed on the surface of the PDMS, diffraction grating side down, such that the piece remained suspended on the surface. This was done with extra care to prevent air bubbles being trapped under the CD piece. The Petri dish and PDMS solution were then allowed to set for $24 \mathrm{~h}$ at $75^{\circ} \mathrm{C}$. After cooling the gel down to room temperature, the CD piece was carefully removed from the PDMS surface and the diffraction pattern could be seen on the PDMS surface. The pattern on the gel surface was checked using an optical reflecting microscope (Zeiss A.1 Axioscope) and compared to the surface of a CD.

PDMS has a hydrophobic surface which makes the cell adhesion process more challenging. The diffraction pattern also has very low visibility in water making it impractical to detect. To increase the hydrophilicity and visibility of the PDMS surface, the gel was coated with a thin platinum layer using a JEOL JFC-1600 sputter coater. The coating time for each sample was $100 \mathrm{~s}$ with a current of $10 \mathrm{~mA}$. The thickness of this layer was $5 \mathrm{~nm}$ based on the machine specifications. The increase in hydrophilicity was verified using static water droplet contact angle measurements. Briefly, the substrate was placed on the platform of the contact angle apparatus SImage Entry 5 (Excimer Inc.) and a $5 \mu$ droplet of water was pipetted onto the surface. A profile image of the droplet was taken and the contact angle was determined by the SImageV05 software (Excimer Inc.). This was repeated at least five times for each sample to obtain an average value for the static contact angle.

\section{Characterization of gel surface}

The surfaces of an unused CD surface, a (1:10) diffraction grating gel, a (1 :35) diffraction grating gel, and the CD surfaces used to 


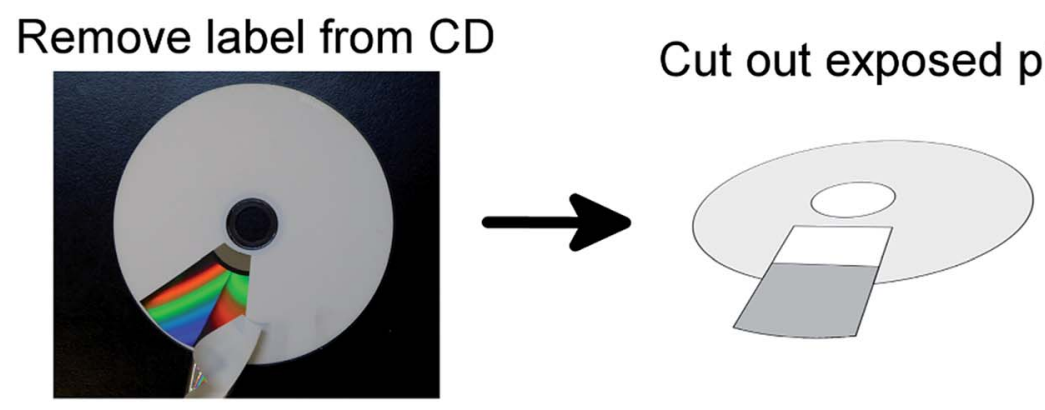

\section{Place piece on PDMS solution}

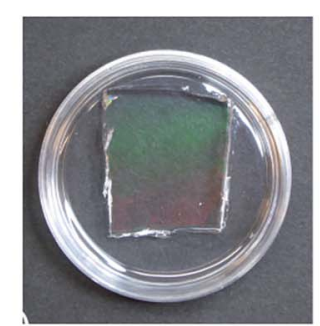

Remove CD piece

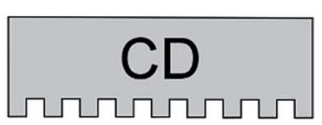

Thermally set PDMS

\section{PDMS Diffraction Grating}

Fig. 2 Outline scheme for preparing PDMS diffraction grating. Plain CD-Rs are used as a template for preparing PDMS diffraction gratings. The label of the CD is removed to expose the underlying diffraction pattern. A piece is cut from the CD and placed on the PDMS solution which is thermally set. The CD piece is then removed and a PDMS diffraction grating is obtained.

mold the patterns were imaged by SEM (Hitachi SU8000). The depth profile of a $(1: 10)$ PDMS grating and a $(1: 35)$ PDMS grating were measured by AFM (JPK NanoWizard III) in tapping mode. The diffraction grating properties were measured using a laser with the purpose of determining the grating element separation, $d$. The $d$ values of a CD piece and (1:35) PDMS grating were measured using a YAG laser where the green light beam (532 nm) was isolated using a quartz Pellin-Broca prism. The apparatus was set up as shown in Fig. 3. The value of $d$ is related to the incident angle, $\theta_{i}$, and the diffracted angle, $\theta_{d}$, through the diffraction equation. By considering only the first order diffraction

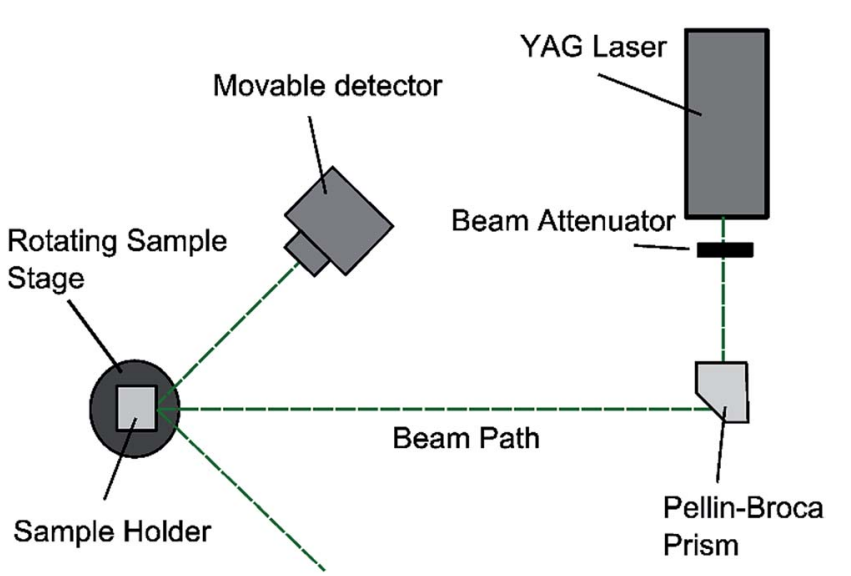

Fig. 3 Laser apparatus for measuring diffraction properties. YAG laser beam is first attenuated by a factor of one hundred through an attenuator. The $532 \mathrm{~nm}$ beam is selected using a Pellin-Broca prism and directed onto the surface of the sample. The sample can rotate to direct the diffracted beams in a desired direction. The angle of rotation can be measured from the stage. The intensity of the beam can be measured using a movable photo-detector. images, $n= \pm 1$, and letting $\theta_{i}=\theta_{d}$, the diffraction equation reduces to $\pm \lambda=2 d \sin \theta_{d}$. The samples were fixed to a rotating stage and rotated from normal incidence until the first order diffracted beam was collinear with the incident beam. The angle that the stage is rotated is recorded as the diffraction angle.

The light intensity of diffracted light from a CD piece, a $(1: 10)$ PDMS gel grating, (1:35) PDMS gel grating, and a platinum coated (1:35) PDMS gel grating were measured using a digital light intensity meter. In each case the sample surface was illuminated by an attenuated YAG laser beam $(532 \mathrm{~nm})$ with $0^{\circ}$ incident angle. The intensity of the diffracted light was measured at a distance of $20 \mathrm{~cm}$ from the sample holder.

\section{Biocompatibility of gel surface}

As there is potential for platinum to have a cytotoxic effect on cardiomyocytes, the biocompatibility of the gel surface and platinum coating was tested. Purified embryonic stem cell derived mouse cardiomyocytes (Cor.At, Axiogenesis, Germany) ${ }^{31}$ were seeded on three 8-well chamber slides (Ibidi \#80826) at $2 \times$ $10^{5}$ cells per well in quadruplicate. The first 8-well slide contained PDMS ( $1: 35)$ with sputter coated platinum (PDMS-PtFn), the second 8-well slide was sputter coated with platinum (Pt-Fn), the final 8-well slide was untreated and acted as a control (Fn). The sputter coated platinum layers were $5 \mathrm{~nm}$ thick. Fibronectin was added to each well to a density of $1.5 \mu \mathrm{g} \mathrm{cm}^{-2}$. The Cor.At cells were seeded on each of the prepared surfaces and cultured according to the manufacturer's protocol. ${ }^{32}$ A CellTiter-Glo 2.0 assay was performed on day two and day eight of the culture. ${ }^{33}$ Briefly, the culture mediums were replaced with $100 \mu \mathrm{l}$ of PBS and $100 \mu \mathrm{l}$ of CellTiter-Glo 2.0 reagent was added. PBS and the reagent were mixed in an empty well without cells and treated as a blank control. After 
incubating for 10 minutes, $50 \mu$ of the lysate was transferred to an opaque 96-well plate and analyzed with a GloMax Microplate Luminometer (Promega). This process was performed for a total of eight measurements per condition and analyzed with ANOVA for detecting the difference among the conditions.

\section{Preparing diffraction gel surface for cell seeding}

The PDMS gratings were coated with fibronectin to promote cell adhesion. For each sample, the surface was irradiated under a laminar flow hood UV light for $30 \mathrm{~min}$. After irradiation the surface was further sterilized with $70 \%$ ethanol and washed twice with DPBS. Next the substrate was coated with fibronectin (Sigma \#F1141) in DPBS with a density of $1.5 \mu \mathrm{g} \mathrm{cm}{ }^{-2}$ for $2 \mathrm{~h}$ at $37{ }^{\circ} \mathrm{C}$. Afterwards the fibronectin solution was removed, the substrate was rinsed with DPBS and $3 \mathrm{ml}$ of culturing medium was added. The substrates were kept in an incubator $\left(37^{\circ} \mathrm{C}\right.$, $5 \% \mathrm{CO}_{2}$ ) until cell seeding.

\section{Isolation and seeding of neonatal rat cardiac cells}

All animal procedures were carried out according to the guidelines for ethical animal experiments provided by Kyoto University (Kyoto, Japan) and the protocol was approved by the iCeMS Animal Experiment Committee (Animal Experiment iCeMS: 18-4). All efforts were made to reduce suffering during the experiments. Sprague-Dawley rats were purchased from Shimizu Laboratory Supplies. For each experiment, ten Sprague-Dawley rat neonates were used. The method for isolating the cardiomyocytes is based on a method used by Iwaki et al. ${ }^{34}$ The pancreatin buffer solutions (one unit for each offspring), and culturing medium were prepared in advance, see Protocol S10† for details. The entire procedure took place under sterile conditions.

Each neonatal rat was placed on ice for $10 \mathrm{~min}$ to anesthetize and induce a low energy state. Each neonate was euthanized by decapitation and the heart ventricles ware rapidly isolated and transferred to a Petri dish containing DPBS. After all ventricles were collected, they were washed in DBPS three times and then minced into a paste using scissors.

Ten millilitres of the digestion enzyme (pancreatin buffer) was added to the ventricle paste. The solution was pipetted into a conical flask and kept stirring in a water bath for $15 \mathrm{~min}$ at $37^{\circ} \mathrm{C}$. The supernatant was removed and discarded. The paste was incubated with $10 \mathrm{ml}$ of fresh digestion enzyme at $37^{\circ} \mathrm{C}$. After $15 \mathrm{~min}$, the supernatant was collected without the solids. The collected solution was centrifuged at $1000 \mathrm{rpm}$ for $4 \mathrm{~min}$. The supernatant was discarded and $4 \mathrm{ml}$ of culture medium was added to the remaining pellet. This suspension was collected in a $50 \mathrm{ml}$ tube and kept at $37^{\circ} \mathrm{C}$. This digestion and collection procedure was repeated 8 times to increase the yield.

After completing the isolation, the cell suspension was seeded on a culture dish for $45 \mathrm{~min}$ at $37^{\circ} \mathrm{C}$ to remove the noncardiomyocyte cells. The floating cells were then collected, centrifuged, counted and adjusted to $3.04 \times 10^{4}$ cells per $\mathrm{ml}$ with culture medium. The PDMS grating samples were prewarmed in the incubator. For each PDMS sample, $300 \mu \mathrm{l}$ of cell suspension was added. For control experiments, cells were also seeded on Elastically Supported Surface (ESS) Petri dishes (Ibidi, Catalogue number \#81199). The Petri dishes have a polymer coating with a specific Young's modulus adjusted to $1.5 \mathrm{kPa}, 15 \mathrm{kPa}$ and $28 \mathrm{kPa}$. In parallel with the PDMS gels, these plates were coated with fibronectin with a density of $1.5 \mu \mathrm{g} \mathrm{cm}^{-2}$ and left for $2 \mathrm{~h}$ at $37^{\circ} \mathrm{C}$.

\section{Observation of cells}

Over the course of several days each sample was observed using an inverted microscope (Nikon Eclipse TS100) to measure the beating frequency of the cells and monitor their overall health. To see the diffracted light, samples were also observed under a stereo microscope (Zeiss SteREO Discovery.V20). Diffracted light can only be observed from an angle; for this reason an external adjustable lamp was set up to illuminate the samples from a chosen direction.

To check that the PDMS grating substrate can be used to monitor changes in beating patterns, epinephrine (Tomogen) was added to the cell culture. Epinephrine, also known as adrenaline, is a hormone well known for its role in an animal's fight-or-flight response. It is also used as a drug, and one of its pharmacological effects is increasing the beating rate of cardiomyocytes. $^{35,36}$ On the fourth day of observation, a PDMS diffraction gel sample with cells beating on the surface was selected and epinephrine solution was added to make a $1 \mu \mathrm{M}$ environment for the cells. Videos were taken immediately before and after the addition of epinephrine using a stereo microscope.

The recorded video was processed to retrieve the beating pattern of the cells. This is accomplished by calculating the average greyscale value, $L$, for each frame of the videos using

$$
L=\frac{\sum_{\text {pixels }}(r+g+b)}{3 N}
$$

where $r, g$, and $b$ are the red, green, and blue intensity values of a pixel respectively, and the sum is taken over all relevant pixels of which there are $N$. The beating pattern is then observed by plotting $N$ against time. The image processing was performed in ImageJ. ${ }^{37}$

\section{Results and discussion}

\section{Preparation of PDMS grating and surface characterization}

Polydimethylsiloxane (PDMS) is a commonly used polymer and can be easily molded into desired patterns. PDMS can be coated with extra-cellular matrix proteins to facilitate cell adhesion ${ }^{38}$ and has been shown to be a suitable substrate for cultivating a number of cardiac cell types including cardiomyocytes from rats $^{15,39}$ and those derived from human pluripotent stem cells (hPSC-CMs). ${ }^{40}$ In this study, PDMS solution was used to make a soft diffraction grating using a CD-R piece as a template. Blank CDs provide a cost effective, reusable, and convenient mold for making diffraction gratings.

The diffraction grating structure could be readily observed under the reflection microscope, Fig. 4. The images confirm that the diffraction pattern of the CD had been copied to the 


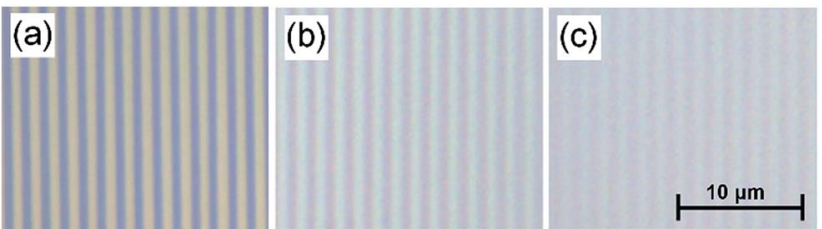

Fig. 4 Microscopic image of surface structure of CD and PDMS gratings. The diffraction pattern of the (a) CD and PDMS gratings are visible under the microscope. The mixing ratios of the PDMS gels are (b) $1: 35$, and (c) $1: 50$. The softer gel $(1: 50)$ produces a lower contrast pattern.

PDMS gel and the samples kept the same spacing pattern as CDR piece, Fig. 4(a). The difference in optical quality between the grating patterns can be easily seen. For the PDMS gratings the quality depends on the mixing ratio of the gels where softer gels have lower optical quality. Gels with mixing ratios of $1: 35$ and $1: 50$ are shown in Fig. 4(b) and (c) respectively, one can see that the pattern is clearly printed on the $(1: 35)$ PDMS gel and the contrast is slightly reduced but still visible on the $(1: 50)$ gel.

The detailed structure of the patterned PDMS can be observed from the SEM imaging. The surface of a $(1: 10)$ PDMS grating, Fig. 5(a), shows a clear grating structure and verifies that the pattern was reliably copied from the template. The surface of the CD piece used to make the grating, Fig. 5(b), shows no sign of damage or tearing from the PDMS. The surface of a softer $(1: 35)$ PDMS gel, Fig. 5(c), also shows a grating structure, though there is some visible deformation of the surface. The CD used to prepare the $(1: 35)$ PDMS, Fig. 5(d), does not show significant signs of tearing or removal.

The AFM depth profile of $(1: 10)$ and $(1: 35)$ PDMS gratings verifies the structure as shown in the SEM, Fig. 6 . The depth

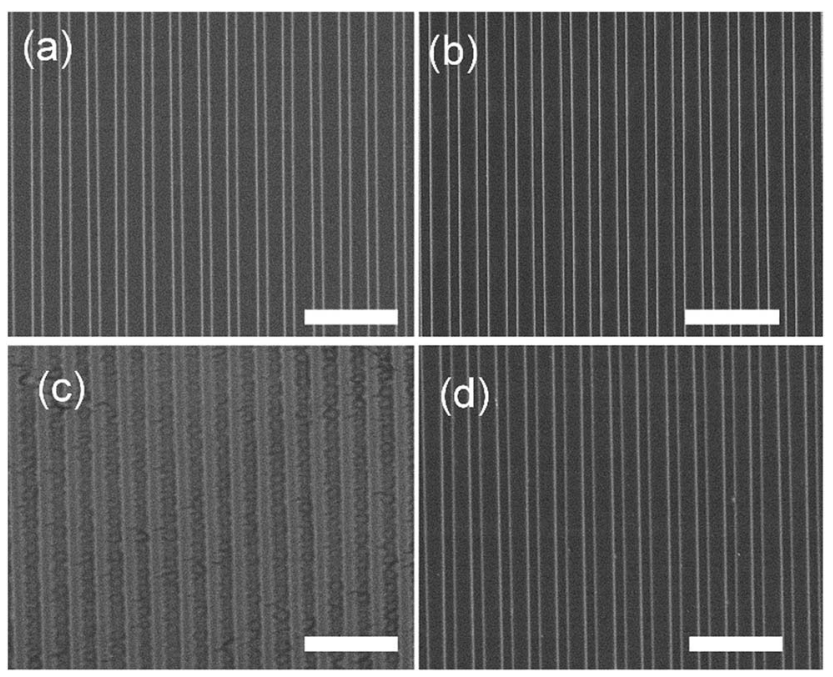

Fig. 5 SEM images of PDMS gratings. SEM of (a) (1:10) PDMS grating surface, (b) CD piece used to prepare (1:10) PDMS grating, (c) $(1: 35)$ PDMS surface, and (d) CD piece used to prepare (1:35) PDMS grating. The $(1: 10)$ PDMS (a) is copied with high fidelity when compared to the CD template. The $(1: 35)$ PDMS (c) copies the CD pattern but with some slight distortion. The scale bar is $5 \mu \mathrm{m}$ in all images.

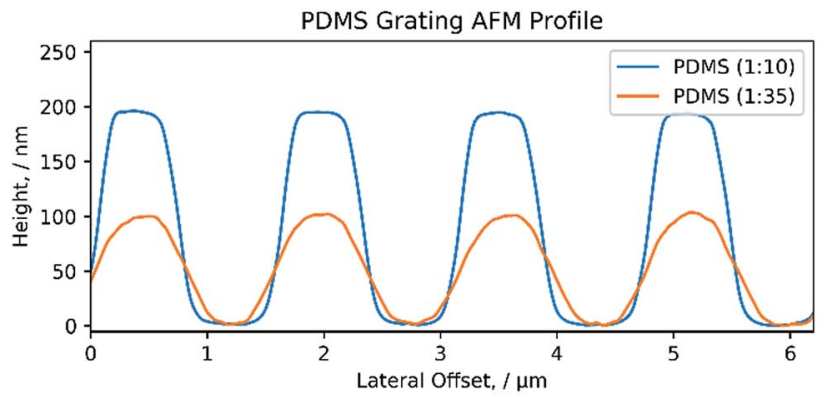

Fig. 6 AFM profile of PDMS grating. Cross-section profile of both a $(1: 10)$ and $(1: 35)$ PDMS diffraction grating.

profiles were obtained by tapping mode AFM and show a consistent depth of $200 \mathrm{~nm}$ and $100 \mathrm{~nm}$ for the $(1: 10)$ and (1:35) PDMS respectively. The difference in height suggests that some of the PDMS could have been removed during the preparation process. Surface AFM images also confirm the condition of the CD and PDMS surfaces as shown by the SEM images in Fig. 5 (ESI S1†).

The SEM and AFM images confirm that high quality diffraction pattern reproductions can be obtained with PDMS. The harder $(1: 10)$ PDMS mixture has the best quality reproduction but the softer $(1: 35)$ PDMS produces a sufficiently high quality pattern for practical use in this study. We found that visible diffraction patterns could reproduced with PDMS (Sylgard 184) up to a $1: 50$ mixing ratio. Beyond this the gel can easily tear during the removal step making reliable reproduction challenging.

The diffraction grating spacing, $d$, was measured using a YAG laser to further confirm that the PDMS faithfully copied the CD diffraction pattern. The $d$ values for the CD-R and $(1: 35)$ PDMS grating were found to be $150 \pm 0.06 \mu \mathrm{m}$ and $1.49 \pm 0.04 \mu \mathrm{m}$ respectively (ESI S2 $\dagger$ ). These values agree with the microscope images and the fact that they are equal confirms that the PDMS can produce a good quality copy of the CD structure. However, the

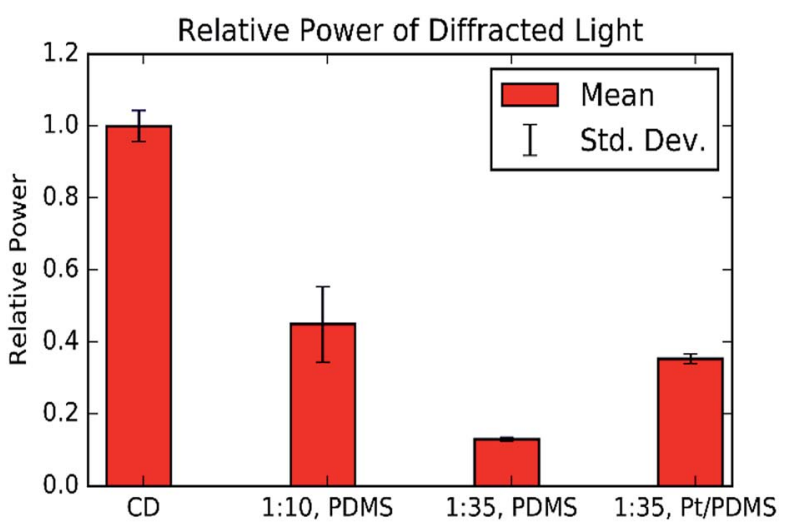

Fig. 7 Power of diffracted light from CD and PDMS samples. The power of the first order diffracted beams is compared between a CD piece, a (1: 10) PDMS grating, a (1: 35) PDMS grating, and a platinum (Pt) coated ( $1: 35)$ PDMS grating. The softer PDMS produces lower power diffracted light, however, the Pt coating appears to restore much of the intensity lost due to the softness of the (1:35) PDMS 


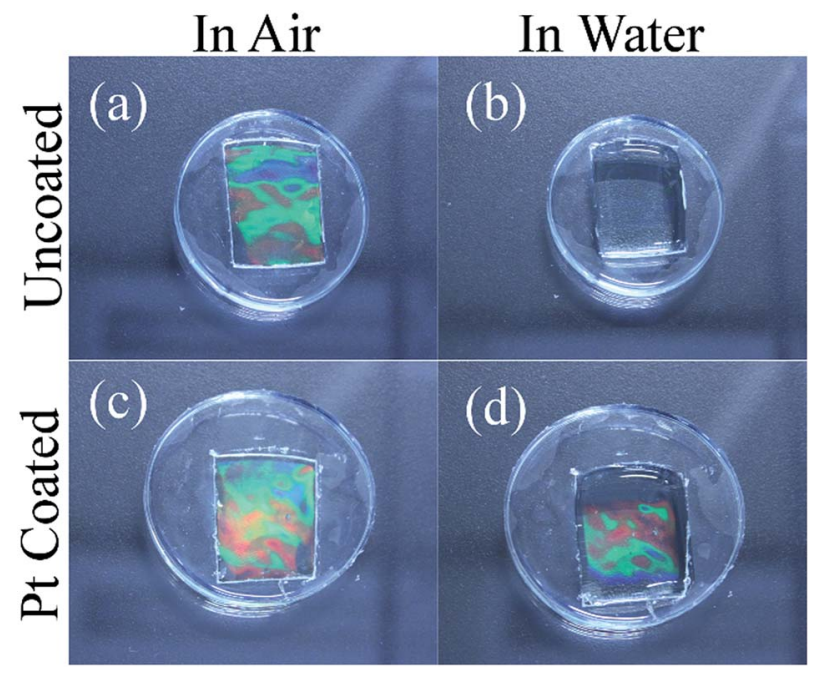

Fig. 8 Diffraction grating gel and effect of platinum coating. PDMS grating when observed (a) in air and (b) in water. Platinum (Pt) coated PDMS grating when observed (c) in air and (d) in water. The Pt layer increases the visibility of the diffraction pattern and allows it to be observed even when submerged. Sylgard 184 PDMS diffraction gratings were prepared with a curing agent/base weight ratio of $1: 35$

laser intensity measurements show that the PDMS gratings have lower reflectivity than the CD-R piece, and the softer PDMS gratings have a lower reflectivity than softer gratings, Fig. 7.

\section{Platinum coating of gel}

To increase the visibility of the PDMS surface, the gel was coated with a thin layer of platinum by sputter coating. The measurements show that the platinum coating can increase the reflection intensity of a PDMS grating, Fig. 7, the results also show that the platinum coating restores the reflecting intensity of the $(1: 35)$ grating to a similar level as the $(1: 10)$ grating. This is beneficial as it increases the effective sensitivity of the substrate and expands the range of useful illumination conditions.

Coating the PDMS gel with platinum has a couple of additional important effects: Without the platinum layer, the refractive indices of PDMS $^{41}(\sim 1.4)$ and water $(\sim 1.3)$ are too similar to produce significant reflection, compare Fig. 8(a) and (b), the platinum layer introduces a larger change in refractive index and allows the diffraction effect to be viewed when the substrate is submerged in an aqueous medium, this comparison is shown in Fig. 8(c) and (d). The second important effect is that the platinum increases the hydrophilicity of the PDMS surface. This was confirmed by static contact angle measurements of water droplets as seen in Fig. 9, where the untreated PDMS surface was found to have an average proceeding contact angle of $118 \pm 4^{\circ}$ and the treated surface had an average contact angle of $83 \pm 15^{\circ}$. The lower contact angle implies that the surface has greater hydrophilicity which is important for improving the adhesion of the cardiac cells to the grating surface.

To demonstrate the same method can be achieved with another metal, PDMS gratings were also coated with gold. Gold is a well-established biocompatible metal, and can be facilely functionalized through thiol chemistry providing opportunities

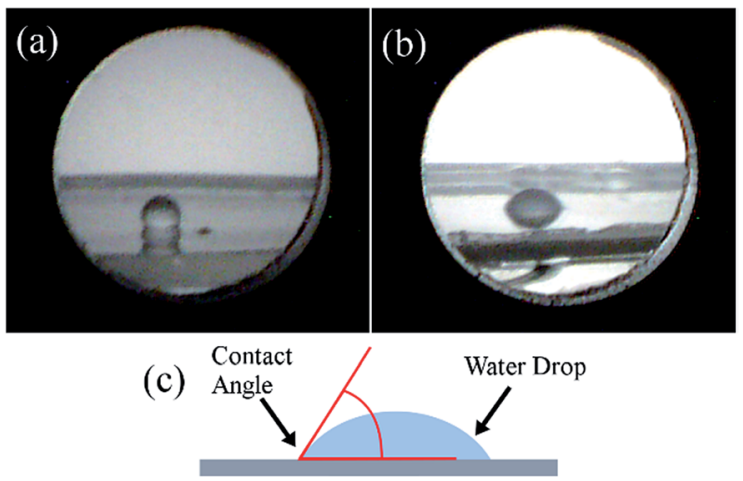

Fig. 9 Contact angle measurements. A $5 \mu$ drop of water is pipetted on the surface of the grating samples and the contact angle is measured with image analysis software. (a) The untreated Sylgard 184 surface, prepared using a ratio of $1: 35$ (curing agent: base) has a contact angle of $118 \pm 4^{\circ}$. (b) After coating the surface with a $5 \mathrm{~nm}$ layer of platinum the contact angle decreases to $83 \pm 15^{\circ}$. The meaning of the contact angle is shown in (c), it is the angle made at the point of contact between a drop of liquid and a surface.

for a wider scope of cellular interactions. Shukla et al. have found that gold nanoparticles do not show cytotoxic effects and are suitable for medical applications. ${ }^{42}$ Hung et al. investigated the biocompatibility of fibronectin coated gold nanoparticles (FNAuNP) with mesenchymal stem cells. ${ }^{43}$ They found the biocompatibility of the FN-AuNP's compared favorably to pure fibronectin. Fendyur and Spira were able to culture rat derived cardiomyocytes on laminin coated gold electrodes. ${ }^{\mathbf{4 4}}$ Such results provide confidence that gold is also a suitable material for our technique. PDMS gratings with a mixing ratio of $1: 10$ and 1:30 were coated with gold using a sputter machine (Quorum Technologies, K575XD Turbo Sputter Coater) with a 1 $\mathrm{mA}$ current for $1 \mathrm{~min}$. After coating the diffraction pattern remained visible while the samples were underwater (ESI S3 $\dagger$ ). Thus gold sputter coating was also found to improve the optical

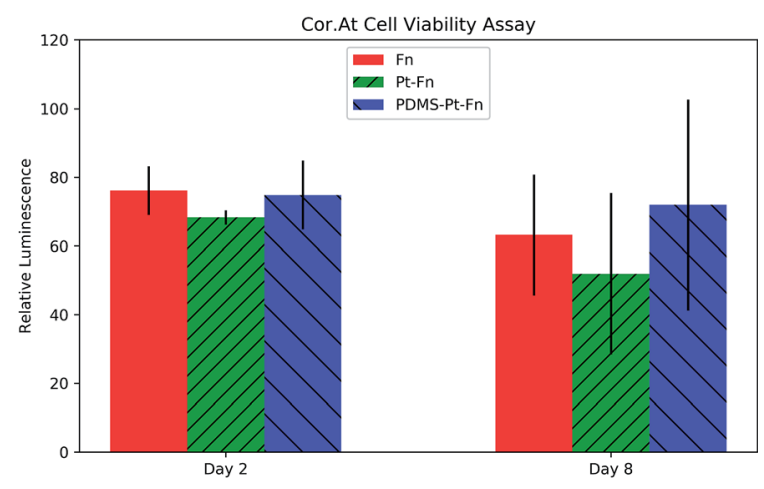

Fig. 10 Cor.At Viability. Cor.At cells were seeded on 8-well plates coated with: platinum and fibronectin (Pt-Fn); PDMS, platinum and fibronectin (PDMS-Pt-Fn); and fibronectin as a control (Fn). After performing the CellTiter-Glo 2.0 assay the luminescence of the medium is proportional to cell quantity. No evidence of toxicity from PDMS or platinum is detected after eight days. Sample luminescence is normalized to an empty well without cells. 


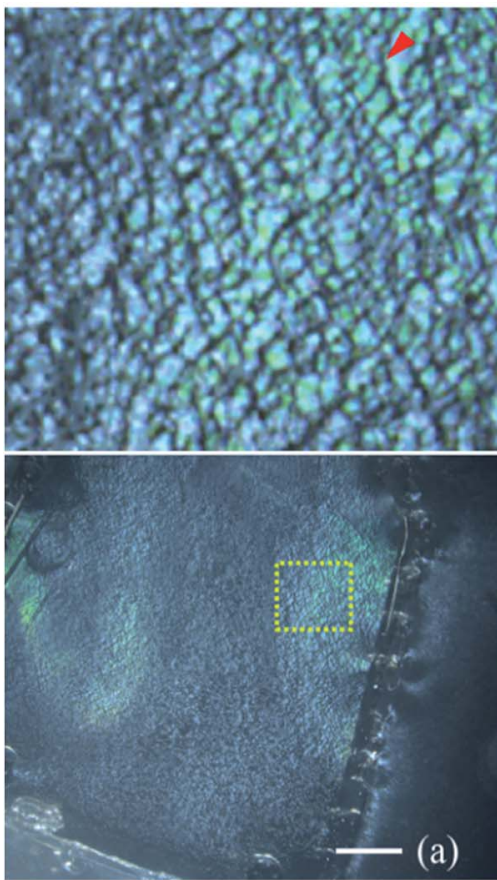

Relaxed Cells

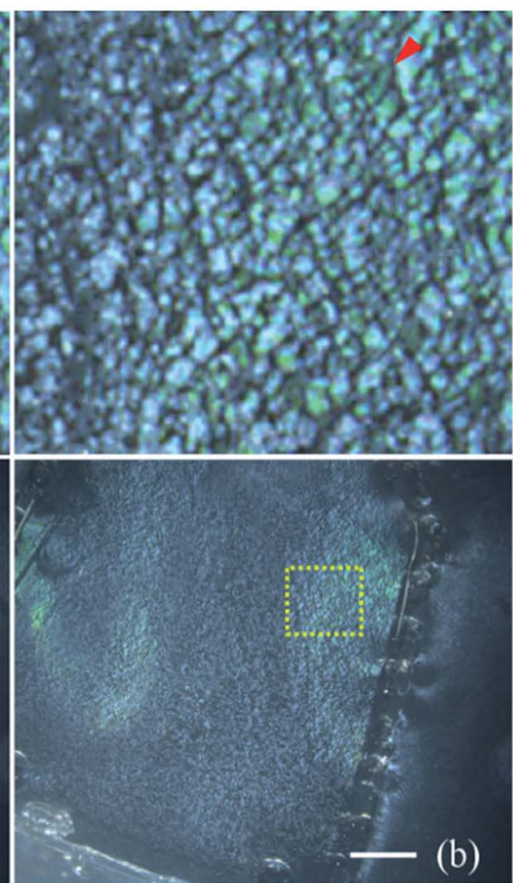

Contracting Cells

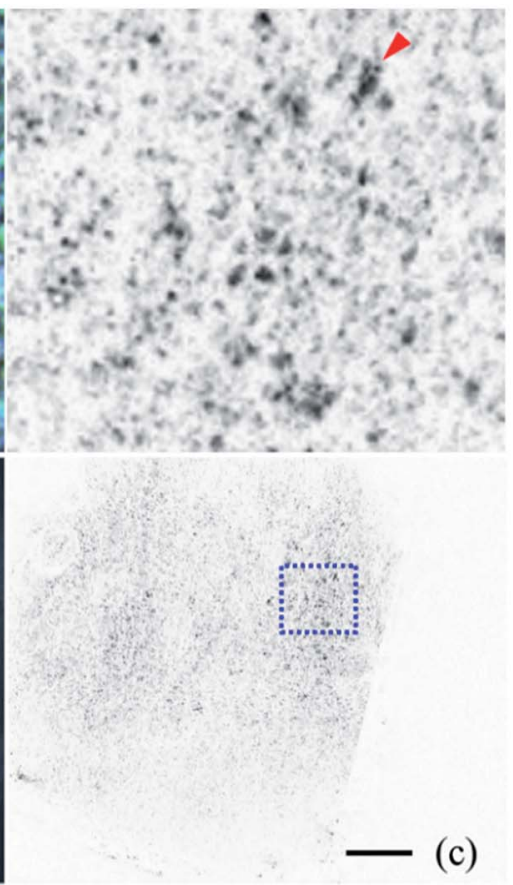

Pixel

Intensity

Difference

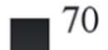

Intensity Difference

Fig. 11 Intensity change between frames of non-beating and beating cardiomyocytes. Images (a) and (b) show the surface of the PDMS diffraction grating during the relaxed and contracting stages of the beat respectively. Image (c) is produced by calculating the difference between the greyscale values of images (a) and (b). Images on the top row show high magnification views of the insets in the bottom row. The red arrow points to an example of a region where there is significant difference in intensity. The image processing was performed in ImageJ followed by an adjustment in contrast to increase the visibility of the image. The frames in this figure are from ESI Video S5. $\dagger$ The scale bars are 2 mm.

quality to a similar level to that obtained from the platinum coated supports.

\section{Biocompatibility of gel surface}

Cor.At cells were seeded in 8-well microslides coated with: PDMS-platinum-fibronectin (PDMS-Pt-Fn), platinum-

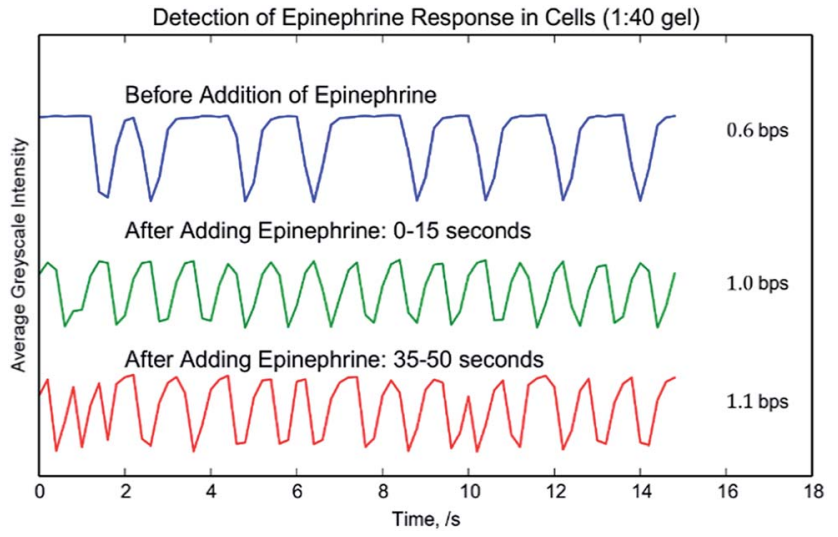

Fig. 12 Cardiomyocyte and epinephrine beating rate. The beating of cardiomyocytes on a diffraction grating substrate was recorded by Video, S8. $\uparrow$ The graph shows the average greyscale value of the video at different times during the experiment. After adding epinephrine the beating rate increases and the beating rate remains consistent over the course of the video. The crosslinking agent: base ratio for the substrate was $1: 40$. fibronectin (Pt-Fn), and fibronectin (Fn). A CellTiter-Glo 2.0 assay was performed on day two and day eight after seeding. The CellTiter-Glo 2.0 assay produces luminescence proportional to the concentration of ATP and is used to quantify metabolically active cells. The results of the assay are shown in Fig. 10. The luminescence of each condition was normalized to the luminescence of a well containing no cells. ANOVA analysis found no significant increase in toxicity with platinum coating, wither on day two $(p=0.12)$ or day eight $(p=0.33)$.

The compatibility of the primary rat cardiomyocytes was checked by seeding cells on the PDMS grating substrates. The cells were monitored over the course of a week by a phase contrast microscope. The primary cardiomyocytes were able to form a confluent, beating layer on each of the PDMS diffraction gels, videos of the cells beating are available as ESI Videos S4(a)-(f).†

\section{Observation of beating cardiomyocytes}

Observation of beating cells was performed under a stereo microscope (Zeiss SteREO Discovery.V20) with illumination from an external lamp at an oblique angle. The diffracted light was observed at various magnifications. At lower magnifications, image distortion could potentially become an issue but no distortion was observed in the $35 \mathrm{~mm}$ culture dishes due to the pattern being centered. Changes in light intensity due to the beating cells were observed and could be seen even at the lowest magnifications, as shown in ESI Video S5. $\dagger$ The change is 
difficult to identify by comparing static images, it can be visualized by calculating the greyscale value difference between video frames at the beginning and end of a contraction, see Fig. 11(a) and (b) respectively. The result of the image difference calculations is shown in Fig. 11(c), with an adjustment of contrast to highlight the changes. The darker regions of the difference image show where the light intensity has changed. This view allows us to see the extent of synchronized beating throughout the confluent layer of cells. Throughout Video S5† we can observe the cardiomyocytes beat in sync, briefly go out of sync, and re-sync towards the end. Further analysis of background noise in the videos and of the variation of signal strength between videos is found in $\mathrm{S} 6 \dagger$ and $\mathrm{S} 7 \dagger$ respectively.

As an application of our cardiomyocyte substrate, epinephrine was added to change the beating rate of the cardiac cells. Epinephrine was added to a cell culture with curing agent:base mixing ratio of $1: 40$. This mixing ratio was chosen due to the stiffness being slightly more optimal for the cells. ${ }^{27,30}$ The response of the cells was recorded and is included as ESI Video S8. $\dagger$ As the video shows, after the epinephrine was added the beating rate of the cells increased on the $(1: 40)$ gel. The beating change is clearly visible from our substrate and the effect can be observed across the entire cardiomyocyte confluent layer.

The observations can be readily extracted through video processing which produces a time-intensity graph for the video, Fig. 12. Clear beating signals can be observed in the charts and we can see that adding epinephrine causes the cells to beat more rapidly immediately after its introduction. The (1:40) sample showed an increase of beating rate from $0.6 \pm 0.2 \mathrm{bps}$ to $1.0 \pm 0.1$ bps. Through peak finding methods, the video processing accurately recorded what we observed in the videos themselves and the technique can be easy applied to an entire batch of videos, supporting any high-throughput applications. Fourier analysis methods can also be used to analyze the beating frequency of the cells. This is demonstrated in $\mathrm{S} 9 \dagger$.

\section{Conclusion}

We report the development of a novel substrate sensor for high-throughput, real-time monitoring of the beating pattern of an entire confluent layer of cardiac cells. This method is non-invasive, requires no additional biological markers, which sets it apart from electrophysiological techniques, but does still place it within context of other visual assessments of cardiomyocyte cell culture. In comparison to those other optical methods, a great advantage of this substrate is that the beating pattern of an entire surface can be viewed at once. Such visual assessments are able to determine various temporal and physical characteristics of the beating cell culture and are easily analyzed by software techniques in a way that allows rapid analysis of many samples. By making use of masking techniques and methods used by Tandon, Marsano, et al., there is potential for modifying the substrate for electrical pacing. ${ }^{45}$ We have demonstrated the principle behind this technique that can be further developed for more sophisticated applications with human cardiac cells and iPSC derived cardiomyocytes.

\section{Conflicts of interest}

There are no conflicts of interest to declare

\section{Acknowledgements}

This work was supported by SPIRITS 2014 of Kyoto University (Supporting Program for Interaction-based Initiative Team Studies) and the iCeMS Accelerated Funding program. E.S. gratefully acknowledges JST-PRESTO (JPMJPR1417). We would like to thank those involved for their support. We would also like to thank Dr Lászlo Köhidai and Livia Polgár from the Chemotaxis Research Group at the Department of Genetics, Semmelweis University, Hungary for their biological assistance, and Prof. Nobuko Naka and Naotaka Yoshikawa from the Department of Physics at Kyoto University for assistance with the laser experiments.

\section{References}

1 Questions and answers on the recommendation to withdraw the marketing authorisations for Clobutinol-containing medicines, EMEA/471052/2007, www.ema.europa.eu/docs/ en_GB/document_library/Referrals_document/clobutinol_107/ WC500094236.pdf, accessed April 20.

2 M. Purhonen, H. Koponen, J. Tiihonen and A. Tanskanen, Outcome of patients after market withdrawal of thioridazine: A retrospective analysis in a nationwide cohort, Pharmacoepidemiol. Drug Saf., 2012, 21, 1227-1231.

3 World Health Organization, WHO Drug Information, 2001, vol. 15.

4 World Health Organization, Pharmaceuticals: Restrictions in Use and Availability, 2001.

5 K. A. Marx, Quartz crystal microbalance: A useful tool for studying thin polymer films and complex biomolecular systems at the solution-surface interface, Biomacromolecules, 2003, 4, 1099-1120.

6 N. Tymchenko, A. Kunze, K. Dahlenborg, S. Svedhem and D. Steel, Acoustical sensing of cardiomyocyte cluster beating, Biochem. Biophys. Res. Commun., 2013, 435, 520-525.

7 A. Kunze, D. Steel, K. Dahlenborg, P. Sartipy and S. Svedhem, Non-Invasive Acoustical sensing of Drug-Induced Effects on the Contractile Machinery of Human Cardiomyocyte Clusters, PLoS One, 2015, 10, e0125540.

8 D. Ossola, et al., Force-Controlled Patch Clamp of Beating Cardiac Cells, Nano Lett., 2015, 15, 1743-1750.

$9 \mathrm{M}$. Dembo and Y. Wang, Stresses at the Cell-to-Substrate Interface during Locomotion of Fibroblasts, Biophys. J., 1999, 76, 2307-2316.

10 A. J. S. Ribeiro, A. K. Denisin, R. E. Wilson and B. L. Pruitt, For whom the cells pull: Hydrogel and micropost devices for measuring traction forces, Methods, 2016, 94, 51-64.

11 A. J. S. Ribeiro, et al., New Methods in Cardiovascular Biology Multi-Imaging Method to Assay the Contractile Mechanical Output of Micropatterned Human iPSC-Derived Cardiac Myocytes, Circ. Res., 2017, 120, 1572-1583. 
12 X. Tang, A. Tofangchi, S. V. Anand and T. A. A. Saif, Novel Cell Traction Force Microscopy to Study Multi-Cellular System, PLoS Comput. Biol., 2014, 10, 1-15.

13 A. W. Feinberg, et al., Muscular thin films for building actuators and powering devices, Science, 2007, 317, 13661370.

14 A. Agarwal, J. A. Goss, A. Cho, M. L. McCain and K. K. Parker, Microfluidic heart on a chip for higher throughput pharmacological studies, Lab Chip, 2013, 13, 3599-3608.

15 A. Grosberg, P. W. Alford, M. L. McCain and K. K. Parker, Ensembles of engineered cardiac tissues for physiological and pharmacological study: Heart on a chip, Lab Chip, 2011, 11, 4165.

16 Y. Tanaka, et al., Demonstration of a PDMS-based biomicroactuator using cultured cardiomyocytes to drive polymer micropillars, Lab Chip, 2006, 6, 230.

17 M. L. Rodriguez, et al., Measuring the contractile forces of human induced pluripotent stem cell-derived cardiomyocytes with arrays of microposts, J. Biomech. Eng., 2014, 136, 51005.

$18 \mathrm{~J}$. You, et al., Cardiomyocyte sensor responsive to changes in physical and chemical environments, J. Biomech., 2014, 47, 400-409.

19 P. Du, C. Cheng, H. Lu and X. Zhang, Investigation of cellular contraction forces in the frequency domain using a PDMS micropillar-based force transducer, J. Microelectromech. Syst., 2013, 22, 44-53.

20 Y. Zhao, C. C. Lim, D. B. Sawyer and R. Liao, Cellular force measurements using single-spaced polymeric microstructures: isolating cells from base substrate, $J$. Micromech. Microeng., 2005, 15, 1649-1656.

21 Y. Zhao and X. Zhang, Cellular mechanics study in cardiac myocytes using PDMS pillars array, Sens. Actuators, A, 2006, 125, 398-404.

$22 \mathrm{~V}$. Chan, et al., Multi-material bio-fabrication of hydrogel cantilevers and actuators with stereolithography, Lab Chip, 2012, 12, 88-98.

23 A. Gaitas, R. Malhotra, T. Li, T. Herron and J. Jalife, A device for rapid and quantitative measurement of cardiac myocyte contractility, Rev. Sci. Instrum., 2015, 86, 34302.

$24 \mathrm{X}$. Zheng and X. Zhang, An optical Moiré technique for cell traction force mapping, J. Micromech. Microeng., 2008, 18, 125006.

25 E. G. Lowen and E. Popov. in Diffraction Gratings and Applications, Marcel Dekker Inc, 1997, vol. 26.

26 L. B. Hazeltine, et al., Effects of Substrate Mechanics on Contractility of Cardiomyocytes Generated from Human Pluripotent Stem Cells, Int. J. Cell Biol., 2012, 2012, 1-13.

27 A. J. Engler, et al., Embryonic cardiomyocytes beat best on a matrix with heart-like elasticity: scar-like rigidity inhibits beating, J. Cell Sci., 2008, 121, 3794-3802.

28 J. G. Jacot, A. D. McCulloch and J. H. Omens, Substrate Stiffness Affects the Functional Maturation of Neonatal Rat Ventricular Myocytes, Biophys. J., 2008, 95, 3479-3487.
29 M. F. Berry, et al., Mesenchymal stem cell injection after myocardial infarction improves myocardial compliance, Am. J. Physiol.: Heart Circ. Physiol., 2006, 290, H2196-H2203.

$30 \mathrm{M}$. Ochsner, et al., Micro-well arrays for 3D shape control and high resolution analysis of single cells, Lab Chip, 2007, 7, 1074-1077.

31 Cor.At Technical Manual, 2012, http://www.veritastk.co.jp/ attached/5769/CorAt_ENG_Manual_v2_2.pdf, accessed: 18th August 2017.

32 Cor.At Cardiomyocytes Seeding on Coverslips, 2012, http:// bio.lonza.com/uploads/tx_mwaxmarketingmaterial/Lonza_ ManualsProductInstructions_Protocol_CorAt_Cardiomyocytes_ Seeding_on_Coverslips.pdf, accessed: 13th August 2017.

33 CellTiter-Glo® 2.0 Assay Technical Manual, https:// www.promega.com/resources/protocols/technical-manuals/ 101/celltiterglo-2-0-assay-protocol/, accessed: 29th October 2017.

34 K. Iwaki, V. Sukhatme, H. Shubeita and K. Chien, Alpha- and Beta-Adrenergic Stimulation Induces Distinct Patterns of Immediate Early Gene Expression in Neonatal Rat Myocardial, J. Biol. Chem., 1989, 265, 13809-13817.

35 Y.-K. Ju and D. G. Allen, How does $\beta$-adrenergic stimulation increase the heart rate? The role of intracellular $\mathrm{Ca}(2+)$ release in amphibian pacemaker cells, Jpn. J. Physiol., 1999, 516, 793-804.

36 Y. Wang, et al., Norepinephrine- and Epinephrine-induced Distinct $\beta 2$-Adrenoceptor Signaling Is Dictated by GRK2 Phosphorylation in Cardiomyocytes, J. Biol. Chem., 2008, 283, 1799-1807.

37 C. A. Schneider, W. S. Rasband and K. W. Eliceiri, NIH Image to ImageJ: 25 years of image analysis, Nat. Methods, 2012, 9, 671-675.

38 N. Annabi, et al., Hydrogel-coated microfluidic channels for cardiomyocyte culture, Lab Chip, 2013, 13, 3569-3577.

39 J. Kim, et al., Quantitative evaluation of cardiomyocyte contractility in a 3D microenvironment, J. Biomech., 2008, 41, 2396-2401.

$40 \mathrm{~A}$. Chen, et al., Integrated platform for functional monitoring of biomimetic heart sheets derived from human pluripotent stem cells, Biomaterials, 2014, 35, 675683.

41 Sylgard 184 Silicone Elastomer Form No. 11-3184B-01, 2014. 42 R. Shukla, et al., Biocompatibility of Gold Nanoparticles and Their Endocytotic Fate Inside the Cellular Compartment: A Microscopic Overview, Langmuir, 2005, 21, 10644-10654.

43 H.-S. Hung, et al., Biocompatibility and Favorable Response of Mesenchymal Stem Cells on Fibronectin-Gold Nanocomposites, PLoS One, 2013, 8, 1-15.

44 A. Fendyur and M. Spira, Toward on-chip, in-cell recordings from cultured cardiomyocytes by arrays of gold mushroomshaped microelectrodes, Front. Neuroeng., 2012, 5, 21.

45 N. Tandon, et al., Surface-patterned electrode bioreactor for electrical stimulation, Lab Chip, 2010, 10, 692-700. 